\title{
GIANT MAGNETORESISTANCE IN PERMALLOY/COPPER MULTILAYERS
}

\author{
T. LUCiŃski AND F. STOBIECKI \\ Institute of Molecular Physics, Polish Academy of Sciences \\ Smoluchowskiego 17, 60-179 Poznań, Poland
}

The oscillatory behaviour of the giant magnetoresistivity versus $\mathrm{Cu}$ sublayer thickness was observed in $\mathrm{Ni}_{83} \mathrm{Fe}_{17} / \mathrm{Cu}$ multilayers with an oscillation period of $1 \mathrm{~nm}$ independently of buffer layer and substrates used in the experiment. The giant magnetoresistance field sensitivity equal to $0.02 \% / O$ and $0.15 \% / O e$ for the first and the second maximum respectively, were found. It was shown that the giant magnetoresistance effect in our multilayers is not only due to the antiferromagnetic coupling between adjacent permalloy layers but also due to the superparamagnetic-like behaviour related to the existence of the intermixed interfacial areas.

PACS numbers: 75.70.Cn, 75.30.Jt

The giant magnetoresistance (GMR) effect attracts much attention not only from physics but also from practical application. Magnetic metallic multilayers (Mls) with enhanced magnetoresistance form potential new candidates for magnetoresistance heads for magnetic recording storage devices. Such GMR heads require large changes of resistance at low fields for temperatures at above room temperature. Thus, of particular application interest are Mls containing magnetically soft permalloy $\left(\left(\mathrm{Ni}_{83} \mathrm{Fe}_{17}=\mathrm{Py}\right)\right.$ layers.

The present paper describes the systematic study of GMR ratio and saturation fields $\left(H_{\mathrm{S}}\right)$ of $\mathrm{Py} / \mathrm{Cu} \mathrm{Mls}$ on $\mathrm{Cu}$ and Py sublayer thicknesses (denoted by $d_{\mathrm{Cu}}$ and $d_{\mathrm{Py}}$, respectively) and on the number of bilayers stack. The $B+[\mathrm{Py} / \mathrm{Cu}] \cdot N$ multilayers, where $B$ and $N$ denote respectively a buffer layer and a number of $\mathrm{Py} / \mathrm{Cu}$ bilayers, were prepared by double facing target sputtering method. The $\mathrm{Cu}$ and Py seed layers were used as $B$, and $N$ was equal to 25,30,40, and 100 . The Mls were deposited onto $\mathrm{Si}(100)$ wafer and glass substrate. GMR effect was measured at room temperature in a magnetic field up to $25 \mathrm{kOe}$. In this paper the GMR ratio is defined as GMR $=\left[R(H=0)-R\left(H=H_{\max }\right)\right] / R\left(H=H_{\max }\right)$.

Figure 1 represents the GMR dependence on $\mathrm{Cu}$ sublayer thickness. The figure collects the GMR data for different $d_{\mathrm{Py}}\left(0.8 \mathrm{~nm}<d_{\mathrm{Py}}<1.5 \mathrm{~nm}\right), B$ and $N$. One can observe an oscillatory behaviour with a period of about $1 \mathrm{~nm}$ with two well distinguished maxima. Similar oscillations in GMR have been reported by Lenczowski [1] in $\mathrm{Ni}_{80} \mathrm{Fe}_{20} / \mathrm{Cu}$ Mls, however only for $B=20 \mathrm{~nm} \mathrm{Cu}$. From Fig. 1 it is evident that the oscillation period depends neither on $N$ nor $B$ and any kind of substrate used in the present experiment. However, the GMR amplitude was found to depend strongly on $d_{\mathbf{P y}}$. 


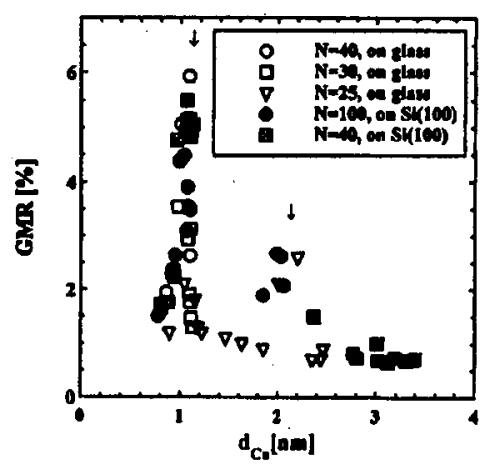

Fig. 1. The GMR versus $\mathrm{Cu}$ sublayer thickness $d_{\mathrm{Cu}}$ for $\mathrm{Py} / \mathrm{Cu}$ Mls deposited onto $\mathrm{Si}(100)$ wafer and glass substrates.

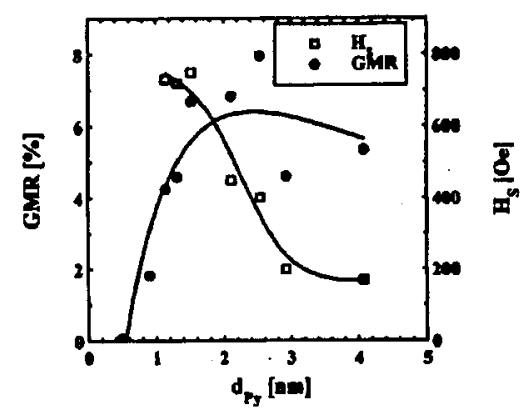

Fig. 2. The GMR ratio and the saturation fields $H_{\mathrm{S}}$ of $\mathrm{Py} / \mathrm{Cu} \mathrm{Mls}$ deposited onto Si wafer with $N=100$, and $B=\mathrm{Cu}\left(20 \mathrm{~nm}\right.$ thick) as a function of $d_{\mathrm{Py}}$ for constant $d_{\mathrm{Cu}}=1 \mathrm{~nm}$.

Figure 2 displays the GMR ratio and the saturation fields of $\mathrm{Py} / \mathrm{Cu} \mathrm{Mls}$ deposited onto Si wafer with $N=100$, and $B=\mathrm{Cu}$ (20 nm thick) as a function of $d_{\mathrm{Py}}$ for constant $d_{\mathrm{Cu}}=1 \mathrm{~nm}$. The highest GMR amplitude of $8 \%$ accompanied by $H_{\mathrm{S}}=400 \mathrm{Oe}$ was found for $d_{\mathrm{Py}}=2.5 \mathrm{~nm}$. For the second maximum in GMR $\left(d_{\mathrm{Cu}}\right)$ the highest GMR amplitude was $3 \%$ while $H_{\mathrm{S}}=20$ Oe. Thus, the field sensitivities of GMR (i.e. $\mathrm{d}(\mathrm{GMR}) / \mathrm{d} H$ ) for the first and the second maximum were $0.02 \% / \mathrm{Oe}$ and $0.15 \% / \mathrm{Oe}$, respectively. Although an interlayer exchange coupling strength $J=2 \times 10^{-5} \mathrm{~J} / \mathrm{m}^{2}$ (for the first maximum in GMR $\left(d_{\mathrm{Cu}}\right)-$ Fig. 1) was found roughly constant independent of $d_{\mathrm{Py}}$, the GMR ratio decreases with decreasing $d_{\mathrm{Py}}$ below $2.5 \mathrm{~nm}$. This can be due to the existence of intermixed region at the $\mathrm{Py} / \mathrm{Cu}$ interfaces caused by mutual solubility of $\mathrm{Ni}$ and $\mathrm{Cu}$. The composition gradient across the $\mathrm{Py} / \mathrm{Cu}$ interface can produce the patchwork of paramagnetic and superparamagnetic areas (i.e. magnetically inactive layer - MIL) together with the ferromagnetic one with reduced magnetization (relative to Py magnetization). The MIL thickness determined from our magnetic moment measurements 

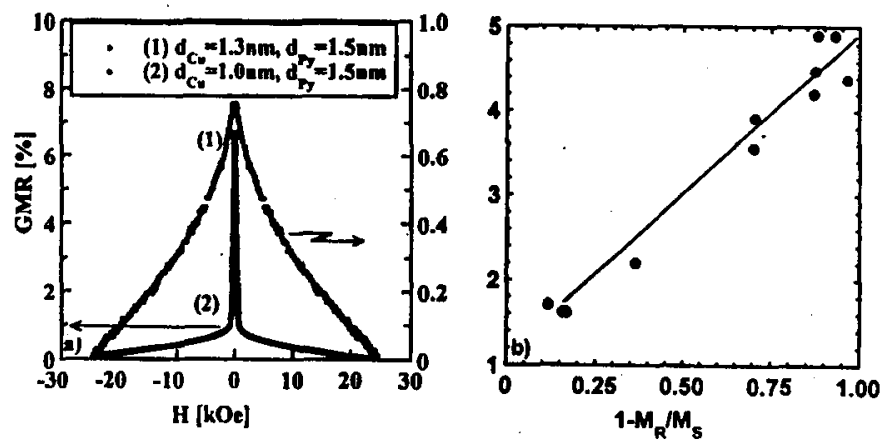

Fig. 3. (a) The GMR $(H)$ dependencies for different $d_{\mathrm{Cu}}$ thicknesses representing the peak and the valley from Fig. 1. (b) The GMR vs. $1-M_{\mathrm{R}} / M_{\mathrm{S}}$ dependence for Mls representing the first maximum in $\operatorname{GMR}\left(d_{\mathrm{Cu}}\right)$.

was found to be of about $0.6 \mathrm{~nm}$ [2]. This value agrees well with $d_{\mathrm{Py}}=0.5 \mathrm{~nm}$ for which GMR $=0$ (Fig. 2).

The influence of such intermixed areas on $\operatorname{GMR}(H)$ is shown in Fig. 3a. Except the low field GMR $(H)$ region exhibiting a well defined saturation field and originating from antiferromagnetic (AF) arrangement of alternating Py layers, a superparamagnetic-like GMR $(H)$ dependence characterised by long unsaturated tails can be observed. The amplitude of the superparamagnetic-like GMR was found to be around $0.8 \%$ independent on $d_{\mathrm{Cu}}$. Thus, the net GMR effect in our $\mathrm{Py} / \mathrm{Cu} \mathrm{Mls}$ has two sources: first due to the AF coupling between adjacent Py layers and second one originating from the intermixed layer at $\mathrm{Py} / \mathrm{Cu}$ interfaces. Due to the strong GMR $\left(d_{\mathrm{Cu}}\right)$ dependence such superparamagnetic-like GMR is more pronounced for Mls with $d_{\mathrm{Cu}}$ out of the $\operatorname{GMR}\left(d_{\mathrm{Cu}}\right)$ peaks. The AF coupled part of the ML can be found from the GMR versus $1-M_{\mathrm{R}} / M_{\mathrm{S}}$ plot, where $M_{\mathrm{R}}$ denotes the remanent magnetization - Fig. $3 \mathrm{~b}$. This figure shows that the Mls representing the first maximum in GMR $\left(d_{\mathrm{Cu}}\right)$ are almost completely AF coupled $\left(1-M_{\mathrm{R}} / M_{\mathrm{S}} \approx 1\right)$, whereas for the Mls representing the valley from Fig. 1 only small fraction of the ML structure can be AF coupled $\left(1-M_{\mathrm{R}} / M_{\mathrm{S}} \approx 0.1\right)$ and $\operatorname{GMR}(H)$ shows in this case superparamagnetic-like field dependence.

Concluding, the oscillatory behaviour of $\operatorname{GMR}\left(d_{\mathrm{Cu}}\right)$ was found in $\mathrm{Py} / \mathrm{Cu}$ multilayers independently on buffer layer and repetition number. GMR amplitudes and $H_{\mathrm{S}}$ were however found to be strongly dependent on $d_{\mathrm{Py}}$. For $d_{\mathrm{Py}}<2.5 \mathrm{~nm}$ this dependence is strongly connected to interfacial interdiffusion processes. The GMR field sensitivity equal to $0.02 \% / O$ and $0.15 \% / O$ for the first and the second maximum respectively, were found.

\section{References}

[1] S.K.J. Lenczowski, Ph.D. Thesis, Eindhoven University of Technology, Eindhoven 1995.

[2] F. Stobiecki, J. Dubowik, T. Luciński, B. Szymański, H. Rohrmann, K. Roell, M. Schmidt, Acta Phys. Pol. A 91, 277 (1997). 